\title{
Effect of various agitation methods on adhesive layer formation of HEMA-free universal dentin adhesive
}

\author{
Ji-Hyun JANG ${ }^{1}$, Bong-Ki JEON², So Yeon $\mathrm{MO}^{2}$, Minjoo PARK², Dongseok CHOI ${ }^{1,3}$, Kyoung-Kyu CHOI \\ and Duck-Su KIM ${ }^{1}$ \\ ${ }^{1}$ Department of Conservative Dentistry, School of Dentistry, Kyung Hee University, Seoul, Korea \\ ${ }^{2}$ Department of Conservative Dentistry, Graduate School, Kyung Hee University, Seoul, Korea \\ ${ }^{3}$ Division of Biostatistics, Oregon Health and Science University-Portland State University of Public Health, Oregon Health and Science University, \\ Portland, OR, USA \\ Corresponding author, Duck-Su KIM; E-mail: dentist96@naver.com
}

\begin{abstract}
This study evaluates the effects of various agitation methods on the adhesive layer formation of a new HEMA-free universal dentin adhesive. The $\mu$ TBS of the universal adhesive, G-Premio BOND in the self-etch mode was evaluated using three agitation methods [passive agitation (PA), active agitation (AA), ultrasonic agitation (UA)], with and without aging treatment. Two-way analysis of variance revealed that aging treatment was not a statistically significant factor. Tukey's HSD test showed significant differences based on the application method, UA>AA>PA. TEM images of the PA group revealed multiple water blisters in the adhesive layers; AA and UA groups presented significantly less or no blisters within the adhesive layers; thus, AA and UA groups exhibited better bonding performance for the HEMA-free universal adhesive. It is assumed that the entrap ped blisters can be reduced with the active application of dentin adhesive, and thus improving the bonding performance.
\end{abstract}

Keywords: Agitation, HEMA, Micro-tensile bond strength, Transmission electron microscopy, Universal dentin adhesive

\section{INTRODUCTION}

Using dentin adhesives with fewer application steps and without technique sensitivity has been a primary goal in the development of dentin adhesives. Incorporating a hydrophilic and hydrophobic component into singlestep self-etch adhesive (SEA) has been introduced in the market. While single-step SEAs have a substantial advantage in that they are simple to use, they demonstrate poor bonding durability because of the increased concentration of hydrophilic monomers ${ }^{1-3)}$. The semi-permeable adhesive layer of single-step SEAs causes disparity between the depth of demineralization and the monomer infiltration, referred to as nanoleakage $e^{4,5}$. There are several clinical suggestions to overcome nanoleakage of single-step SEAs. Prolonged application time ${ }^{6,7)}$ and either active agitation $(\mathrm{AA})^{7-9)}$ or ultrasonic agitation $(\mathrm{UA})^{10-12)}$ improve the chemical interaction with the tooth substrate by dispersing etching by-products into the hybrid layer, thereby enhancing the bonding performance.

In the early 2010s, a universal adhesive system was introduced which was fairly versatile and thus could be used in both etch-and-rinse and self-etch modes ${ }^{13)}$. This system typically comprises 10-methacryloyloxydecyl dihydrogen phosphate (MDP), which contributes to micromechanical interlocking, supplemented by chemical bonding between MDP and hydroxyapatite crystallites, resulting in an MDP-Ca salt ${ }^{14,15}$. Yoshihara et $a l .{ }^{16)}$ first reported the characteristic of self-assembled nanolayering structures at the adhesive interface, which result from the interaction between MDP and hydroxyapatite. Many studies have confirmed that this unique nanolayering structure contributes to the longterm bonding durability of the hybrid layer and protects it from hydrolytic degradation ${ }^{14,17,18)}$.

2-hydroxyethyl methacrylate (HEMA) is usually used to incorporate complex chemical compositions, including hydrophilic and hydrophobic monomers, solvents, water, and additives, within the simplified dentin adhesive bottle ${ }^{19}$. HEMA behaves as a wetting agent and improves the diffusion of dentin adhesives into deep demineralized dentin. It also plays an important role in the inhibition of the phase-separation phenomenon of dentin adhesives ${ }^{20,21}$. Nevertheless, HEMA deteriorates the mechanical properties of polymerized adhesives because of its monomethacrylate chemical structure and negatively influences the hydrolytic degradation of the adhesive layer ${ }^{22,23)}$. Thus, some dentin adhesives exclude HEMA in their composition.

In our study, we evaluated the bonding performance of a HEMA-free universal adhesive for various application methods. The null hypotheses were as follows: (1) there is no effect of the application method on the bond strength of the HEMA-free universal adhesive in the self-etch mode and (2) there is no effect of aging treatment on the bond strength of the HEMA-free universal adhesive in the self-etch mode.

\section{MATERIALS AND METHODS}

\section{Specimen preparation}

Thirty caries-free third molars were used after acquiring informed consent from the donors, as approved by the Institutional Review Board of Kyung Hee University Dental Hospital (KHD IRB 1507-3). The teeth were 
Table 1 Materials used in this study

\begin{tabular}{clll}
\hline \multicolumn{1}{c}{ Product } & \multicolumn{1}{c}{ Manufacturer } & Batch No. & \multicolumn{1}{c}{ Chemical composition } \\
\hline $\begin{array}{l}\text { Dentin adhesive } \\
\text { G-Premio BOND } \\
\text { (GPB) }\end{array}$ & $\begin{array}{l}\text { GC, Milford, } \\
\text { DE, USA }\end{array}$ & 1501152 & $\begin{array}{l}\text { MDP, 4-MET, MEPS methacrylate monomer, } \\
\text { acetone, water, initiator, silica filler }\end{array}$ \\
$\begin{array}{l}\text { Composite resin } \\
\text { Z250 } \\
\text { (shade A2) }\end{array}$ & $\begin{array}{l}\text { 3M ESPE, } \\
\text { St Paul, MN, USA }\end{array}$ & N788047 & $\begin{array}{l}\text { Bis-EMA, UDMA, Bis-GMA, TEGDMA, } \\
\text { silane-treated ceramic filler, benzotriazole, EDMAB }\end{array}$ \\
\hline
\end{tabular}

Bis-GMA: bisphenol-A-diglycidyl methacrylate; TEGDMA: triethyleneglycol dimethacrylate; TCD-di-HEA: tricyclodecaneurethane dimethacrylate; UDMA: urethane dimethacrylate; Bis-EMA: bisphenol-A-polyethylene glycol diether dimethacrylate; EDMAB: ethyl 4-dimethyl aminobenzoate

cleaned and stored in distilled water before preparation. The occlusal enamel was removed, and the flat dentin surface was exposed under water irrigation using a lowspeed diamond saw (ISOMET ${ }^{\mathrm{TM}}$, Buehler, Lake Bluff, IL, USA). Each dentin surface was ground and polished with 320-grit silicon carbide abrasive paper to produce a standard smear layer. The teeth were divided randomly into six groups according to the agitation method and artificial aging treatment.

The chemical compositions of materials used in this study are shown in Table 1. The HEMA-free universal dentin adhesive G-Premio BOND (GPB, GC, Tokyo, Japan) was applied to the dentin surface in the self-etch mode according to the manufacturer's instructions using three different agitation modes: passive agitation (PA), active agitation (AA), and ultrasonic agitation (UA). For the PA mode, GPB was applied and left undisturbed for $10 \mathrm{~s}$ according to the manufacturer's instructions. For the AA mode, GPB was applied with AA for 10 s. For the UA mode, GPB was applied for $10 \mathrm{~s}$ with microbrush applicator (Kerr, Orange, CA, USA), which the shank of microbrush is continuously touched while its application with a tip of ultrasonic instrument (Piezon Master 400, EMS, Le Sentier, Switzerland) set at a frequency of 27$30 \mathrm{kHz}$. The applied adhesive was dried for $5 \mathrm{~s}$ followed by light-curing for $20 \mathrm{~s}$ using a light-emitting diode lightcuring unit (Bluephase 20i, Ivoclar Vivadent, Schaan, Liechtenstein) with a light intensity of $700 \mathrm{~mW} / \mathrm{cm}^{2}$ for each experimental group. Then, $4 \mathrm{~mm}$ of a composite resin (Z250, 3M ESPE, St. Paul, MN, USA) was layered in increments of $2 \mathrm{~mm}$. Each composite layer was lightcured for $40 \mathrm{~s}$. The specimens were stored in distilled water at $37^{\circ} \mathrm{C}$ for $24 \mathrm{~h}$.

\footnotetext{
Micro-tensile bond strength test and fracture mode analysis

After $24 \mathrm{~h}$ of storage, the specimens were sectioned in a direction perpendicular to the adhesive surface using a water-cooled high-speed diamond saw (ISOMET 5000, Buehler). Approximately ten to eleven compositedentin beams were acquired, and among them, six central beams were selected from each tooth. Total of 180 composite-dentin beams with a surface area of $1 \times 1$ $\mathrm{mm}^{2}$ were prepared for the microtensile bond strength ( $\mu$ TBS) test.
}

Specimens were equally divided into two sub-groups according to the artificial aging treatment: immediate (PAI, AAI, UAI) and aging treatment (PAA, AAA, UAA) groups. For the immediate groups, the specimens were fixed to a testing jig with a cyanoacrylate adhesive (ZAPIT, Dental Ventures of America, Corona, CA, USA) and the $\mu$ TBS test was performed using a testing machine (Micro Tensile Tester, BISCO, Schaumburg, IL, USA) at a crosshead speed of $0.5 \mathrm{~mm} / \mathrm{min}$. For the aging treatment groups, the beams were immersed in $10 \%$ aqueous sodium hypochlorite solution for $1 \mathrm{~h}$ at room temperature, and the $\mu$ TBS test was performed as above.

The results of the $\mu$ TBS test were statistically analyzed using two-way analysis of variance (ANOVA) and Tukey's honest significant difference (HSD) post hoc test $(a=0.05)$ to determine the effects of application modality versus aging treatment and the interaction between these factors. Statistical analyses were performed using SPSS for Windows (version 12.0, IBM SPSS, Chicago, IL, USA).

Failure modes were observed under a stereo microscope at magnifications of up to $\times 40$. The failure modes of the specimens were classified as follows: adhesive failure at the resin/dentin interface (A), cohesive failure within the composite resin (CC), cohesive failure within dentin (CD), and mixed failure at the resin/dentin interface including cohesive failure of the neighboring substrate (M).

\section{Transmission electron microscopy (TEM)}

Bonded interfaces were morphologically assessed by TEM. TEM specimens were prepared according to the method suggested by Tay et al. ${ }^{24)}$. In each experimental group, two additional mandibular third molars were selected. The specimens were prepared concurrently with the $\mu$ TBS tests. Two central slabs were sectioned from each tooth, yielding four slabs in total. Two coats of nail varnish were applied to the slabs, leaving $1 \mathrm{~mm}$ between the varnish and the adhesive interface. The specimens were immersed in $50 \mathrm{wt} \%$ ammoniacal silver nitrate solution ( $\mathrm{pH}$ 9.5) for $24 \mathrm{~h}$ in a dark room; they were then irrigated using distilled water and stored in a developing solution for $8 \mathrm{~h}$ under fluorescent light, during which time they were irrigated using distilled 
water.

The specimens were fixed in Karnovsky's solution (2.5\% glutaraldehyde and $2 \%$ paraformaldehyde in 0.1 $\mathrm{M}$ sodium phosphate buffer [pH 7.4]) for $8 \mathrm{~h}$, and then washed for $3 \times 10 \mathrm{~min}$ using $0.1 \mathrm{M}$ sodium phosphate buffer. Post-fixation was performed using a 1\% osmium tetroxide solution for $2 \mathrm{~h}$. Subsequently, each specimen was washed for $3 \times 10$ min each. The post-fixed specimens were dehydrated in ascending grades of ethanol (from 50 to $100 \%$ ) for $10 \mathrm{~min}$ each, and embedded in an epoxy resin. After screening a $200 \mathrm{~nm}$ semi-thin section of each specimen using an ultramicrotome equipped with a diamond knife, an approximately $70 \mathrm{~nm}$ ultra-thin section was sliced and collected on a 75 mesh formvarcoated copper grid. After drying, the samples were examined using TEM (JEM-1010 TM, JEOL, Tokyo, Japan) at $100 \mathrm{kV}$.

\section{RESULTS}

The overall $\mu$ TBS and failure mode in all experimental groups are shown in Table 2. Two-way ANOVA revealed that the main factor, aging treatment, was not statistically significant $(\mathrm{F}=0.172, p=0.68)$. The other main factor, application modality, showed a statistically significant difference $(\mathrm{F}=56.93, p<0.01)$. There was a significant interaction between the application method and the aging treatment $(\mathrm{F}=3.46, p=0.33)$. Tukey's HSD test showed significant differences according to the application method, in the following order: UA $>$ AA $>$ PA $(p<0.01)$. The experimental groups did not show a significant difference according to the aging treatment in each application method. In failure mode distribution, an adhesive failure mode was predominant, which ranged from 90 to $100 \%$ regardless of the application method or aging treatment.

TEM images of the PA group revealed multiple entrapped water blisters in the adhesive layer and some tiny droplets adjacent to the hybrid layer (Fig. 1). Compared to TEM images of the specimens from the
PA groups, those of the specimens from the AA and UA groups revealed significantly less or no blisters within

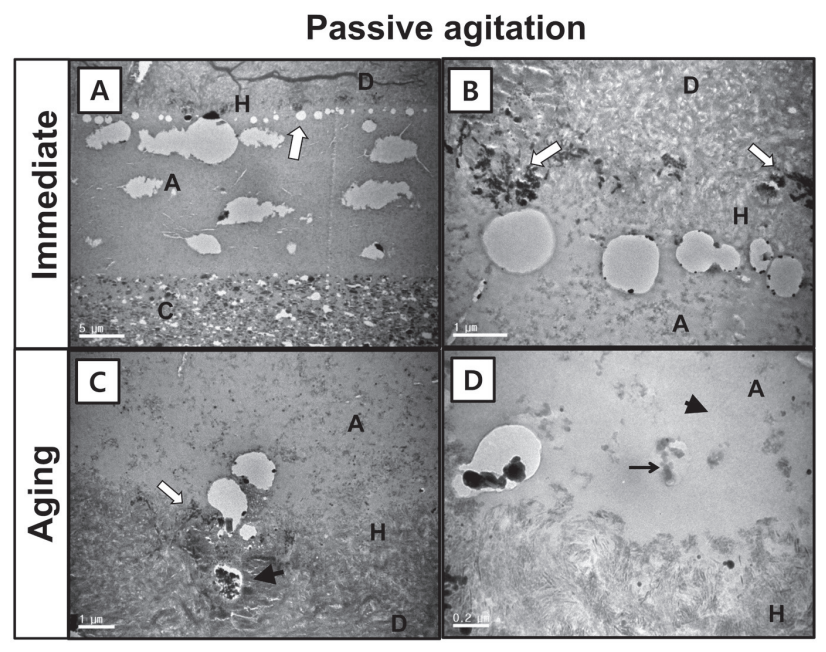

Fig. 1 TEM images of the PA groups subdivided according to the aging treatment: immediate $(\mathrm{A}, \mathrm{B})$ or aging (C, D) treatment group.

(A) A thin hybrid layer of approximately $1 \mu \mathrm{m}$ was observed. There were multiple entrapped blisters of various sizes and shapes within the adhesive layer. Note the higher distribution of blisters close to the bottom of the adhesive layer. A number of tiny droplets (arrow) were observed adjacent to the hybrid layer. (B) High magnification of the hybrid layer. Demineralized hybrid layer was silver stained (arrow). (C) Silver nitrate infiltration was detected in the hybrid layer (arrow) and blisters (black arrow head). (D) Collagen fibrils were partially dissolved in the adhesive layer and seen as an electrolucent area (black arrow head). The black arrow indicated that the infiltrated adhesive resin protected the collagen fibrils from sodium hypochlorite aging treatment. A, adhesive layer; C, composite resin; D, dentin; H, hybrid layer

Table 2 Microtensile bond strength values and fracture mode of experimental groups $(n=30)$

\begin{tabular}{|c|c|c|c|c|c|c|}
\hline \multirow{3}{*}{$\begin{array}{l}\text { Agitation } \\
\text { method }\end{array}$} & \multirow{3}{*}{$\begin{array}{c}\text { Aging } \\
\text { treatment }\end{array}$} & \multirow{3}{*}{$\mu \mathrm{TBS}(\mathrm{MPa})$} & \multicolumn{4}{|c|}{ Fracture mode } \\
\hline & & & \multirow{2}{*}{ Adhesive } & \multicolumn{2}{|c|}{ Cohesive } & \multirow{2}{*}{ Mixed } \\
\hline & & & & In composite & In dentin & \\
\hline \multirow{2}{*}{ PA } & Immediate & $23.5 \pm 4.97^{\mathrm{a}}$ & 28 & 2 & 0 & 0 \\
\hline & Aging & $22.3 \pm 3.16^{\mathrm{a}}$ & 30 & 0 & 0 & 0 \\
\hline \multirow{2}{*}{$\mathrm{AA}$} & Immediate & $27.9 \pm 5.71^{\mathrm{b}}$ & 28 & 2 & 0 & 0 \\
\hline & Aging & $25.4 \pm 5.05^{\mathrm{b}}$ & 28 & 0 & 2 & 0 \\
\hline \multirow{2}{*}{ UA } & Immediate & $33.1 \pm 5.54^{\mathrm{c}}$ & 28 & 1 & 1 & 0 \\
\hline & Aging & $35.4 \pm 6.00^{\mathrm{c}}$ & 27 & 1 & 1 & 1 \\
\hline
\end{tabular}

Mean \pm standard deviation.

Different letters indicate differences in statistical significance among groups (Tukey's post hoc test, $\alpha=0.05$ )

PA, passive application; AA, active agitation; UA, ultrasonic agitation; A, adhesive failure at resin/dentin interface; C-R, cohesive failure within composite resin, C-D, cohesive failure within dentin; M, mixed failure at resin/dentin interface. 


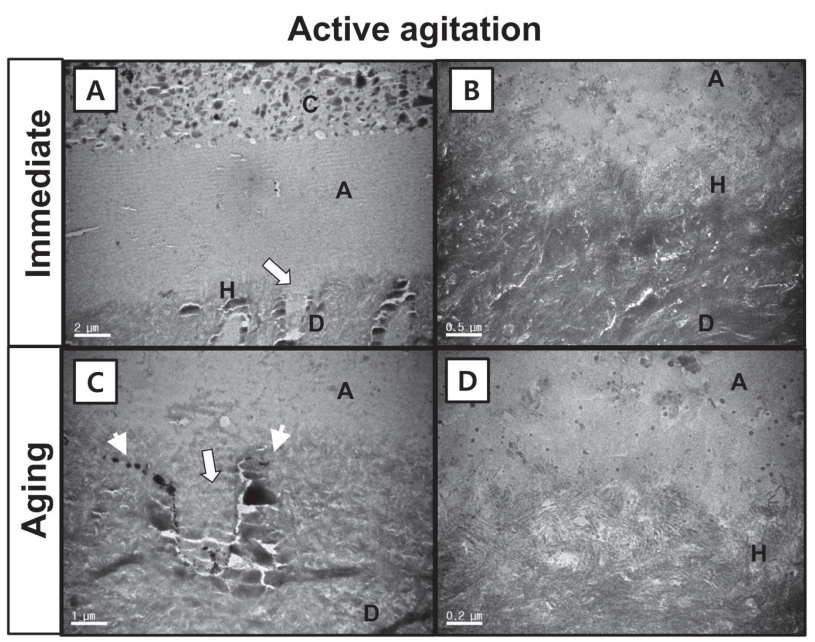

Fig. 2 TEM images of the AA groups subdivided according to the aging treatment: immediate $(\mathrm{A}, \mathrm{B})$ or aging (C, D) treatment group.

(A) Hybrid layer thickness is within $1 \mu \mathrm{m}$. Resin tags (white arrow) were rarely formed along the dentinal tubule. There were significantly less or no blisters within the adhesive layer of the AA group compared to those observed in the TEM images of the PA group shown in Fig. 1. (B, D) Electrodense area was detected on the hybrid layer, which was protected by collagen fibrils. (C) Silver nitrate infiltration (arrow head) indicated nanoleakage in the aging treatment specimen. Silver stains were observed in the bottom layer of the hybrid layer. $\mathrm{A}$, adhesive layer; $\mathrm{C}$, composite resin; $\mathrm{D}$, dentin; $\mathrm{H}$, hybrid layer

the adhesive layers (Fig. 2 and Fig. 3, respectively).

A relatively thin hybrid layer of approximately 1 $\mu \mathrm{m}$ was commonly observed in all experimental groups. The hybrid layer with sparse resin tags and partially demineralized hydroxyapatite revealed the typical microscopic image of the adhesive layer produced by the self-etch dentin adhesive. Silver nitrate infiltration, which is indicative of nanoleakage, was observed in the aging treatment specimens. Silver stains were observed in the bottom layer of the hybrid layer.

\section{DISCUSSION}

In this study, we evaluated the bonding performance of a HEMA-free universal adhesive, GPB, in the self-etch mode with three different application methods. The main finding was that AA or UA application provided better bonding performance than PA application; thus, the first null hypothesis was rejected. Moreover, aging treatment had no effect on the bonding performance within the same application method group; thus, the second null hypothesis was accepted.

There have been many in vitro studies regarding the application of simplified self-etch dentin adhesives to offset limitations with respect to bonding performance.

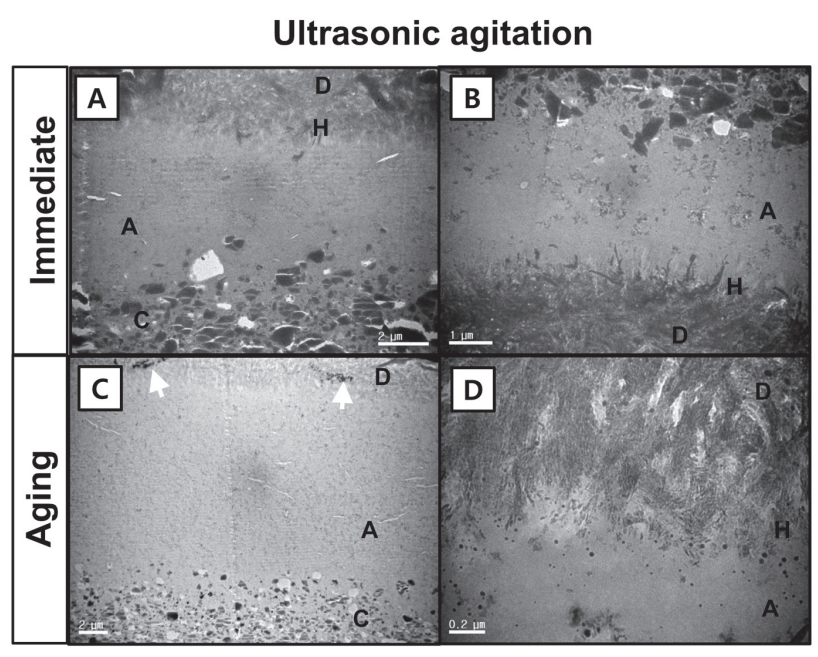

Fig. 3 TEM images of the UA groups subdivided according to the aging treatment: immediate $(\mathrm{A}, \mathrm{B})$ or aging (C, D) treatment group.

(A) There were significantly less or no blisters within the adhesive layer of the UA group compared to those observed in the TEM images of the PA group shown in Fig. 1. (B, D) Electrodense area was detected in the hybrid layer, which was protected by collagen fibrils. (C) Silver stains (arrow head) were sparsely observed in the bottom layer of the adhesive layer, indicating nanoleakage. A, adhesive layer; C, composite resin; D, dentin; H, hybrid layer

Miyazaki et al. reported that AA application improves the bond strength of a dentin primer with a threestep etch-and-rinse dentin adhesive compared to PA application ${ }^{9}$. The authors explained that the improved bonding performance due to AA application originated from the increased penetration of the acidic primer into the etched dentin, which enhanced the micromechanical interlocking and chemical interaction. Amaral et al. ${ }^{8)}$ suggested that the vigorous AA application mode of single-step SEAs enhances bond strength because it facilitates monomer diffusion and solvent evaporation. UA application was also demonstrated to improve the bond strength of both etch-and-rinse ${ }^{10)}$ and self-etch ${ }^{11)}$ dentin adhesives for dentin using ultrasonic vibrational energy. Application of UA energy could induce acoustic streaming and increase infiltration of the dentin adhesive into the dentinal structure. To the best of our knowledge, there have been no studies that have simultaneously compared the bonding effectiveness of three application modalities, UA, AA, and PA. Our results demonstrated that the UA application mode is the most effective for increasing the bond strength in all experimental groups, regardless of aging treatment. Applying ultrasonic energy during agitation application is an effective clinical technique for improving bonding. However, ultrasonic vibration can be cumbersome and requires an additional device. Fortunately, agitation application still plays an important role in improving bond strength, 
compared to PA application of adhesives, corresponding with the results of previous studies considering other types of dentin adhesive systems ${ }^{8,9)}$. Considering the difficult clinical application and the necessity of an ultrasonic device for the UA mode, the AA mode may be a more user-friendly alternative application method for the improved bonding performance of dentin adhesives.

Though favorable bonding efficacy of universal adhesives has been reported via in vitro and clinical studies $^{25-27)}$, it is still vulnerable due to the lack of longterm stability of the bonding interface and clinical evaluation. The presence of HEMA in universal adhesives may not influence initial bond strength but may affect long-term bond stability. As previously described, an important characteristic of HEMA is the inhibition of dentin-adhesive phase separation ${ }^{19}$. HEMA functions as a solvent keeping the adhesive ingredients in solution by improving the miscibility of the hydrophobic and hydrophilic components ${ }^{20,21}$. However, it attracts water and dilutes monomers readily, resulting in hydrolytic degradation of the adhesive layers ${ }^{22,23}$. HEMA-free adhesives have been introduced to overcome these disadvantages; however, there are still limitations due to the inclusion of higher concentrations of solvent and water, resulting in more blisters in the adhesive layer due to difficulties in solvent evaporation. In our study, the entrapment of water blisters within the adhesive layer shown in the TEM image of the PA group (Fig. 1) is a representative ultrastructural image of HEMAfree adhesives, as demonstrated in previous studies ${ }^{20,21}$. Interestingly, AA (Fig. 2) and UA (Fig. 3) groups presented well-hybridized adhesive layers with less or no entrapped blisters. Thus, the droplets in the PA groups mostly disappeared after AA or UA method. It was assumed that agitation application of the adhesive might be associated with the removal of multiple blisters in the hybrid layer, which is achieved by the omission of HEMA. Agitation, with or without ultrasonic vibration, was effective for activating monomer diffusion, leading to enhanced bonding performance. The different microscopic morphologies of the adhesive layer might explain the difference in $\mu$ TBS results between the PA group and the AA and UA groups.

In this study, we used the sodium hypochlorite storage method to validate the bond durability of the adhesive interface, and the effect of aging treatment was not significant. Sodium hypochlorite storage accelerates the degradation of the adhesive interface and theoretically deteriorates the stability of the organic component of the hybrid layer ${ }^{28}$. However, in this study, the hybrid layer and underlying dentin substrate were not chemically altered with aging treatment, possibly due to the usage mode of the dentin adhesive. Generally, the hybrid layer is thicker in the etch-and-rinse mode than in the self-etch mode using the same universal adhesive ${ }^{29}$. Moreover, the self-etch dentin adhesive did not eliminate but rather modified the smear layer; thus, the hybrid layer produced from the self-etch mode, as opposed to the etch-and-rinse mode, included more inorganic substrates. In our study, we used the universal adhesive in self-etch mode, and produce a relatively thick standardized smear layer by polishing the dentin surface with 320-grit $\mathrm{SiC}$ abrasive paper instead of 600grit $\mathrm{SiC}$ abrasive paper, so that the smear layer can play a relevant role in the validation of the effect of the agitation application mode. Our results revealed that the artificial aging has no effect for the adhesive interface, and it might be related to the thick smear layer which attributes to minimize the effects of sodium hypochlorite treatment. Though our TEM analysis revealed no effects of the aging treatment, sodium hypochlorite aging method has the limitation because it is too severe and rapid deproteinization compared with the long-term aging treatment. Further long-term real-time bond durability evaluation is needed.

In conclusion, with or without ultrasonic energy, active application improves the bonding performance of HEMA-free universal adhesives in the self-etch mode. It is assumed that AA of dentin adhesive attribute to reduce the entrapped blisters within adhesive layer, which are usually originated from the exclusion of HEMA in its composition. Within limitation of our study, further investigations using different universal adhesives in both self-etch and etch-and-rinse modes and long-term real-time bonding durability evaluation are required, and we believe that it would be valuable to yield a clinically relevant comparison of our study.

\section{ACKNOWLEDGMENTS}

This work was supported by the National Research Foundation of Korea (NRF) grant funded by the Korea government (MSIT, No. 2015R1C1A1A02036729).

\section{CONFLICT OF INTEREST}

The authors declare no conflicts of interest, real or perceived, financial or nonfinancial.

\section{REFERENCES}

1) Peumans M, Kanumilli P, De Munck J, Van Landuyt K, Lambrechts P, Van Meerbeek B. Clinical effectiveness of contemporary adhesives: a systematic review of current clinical trials. Dent Mater 2005; 21: 864-881.

2) Breschi L, Mazzoni A, Ruggeri A, Cadenaro M, Di Lenarda R, De Stefano Dorigo E. Dental adhesion review: aging and stability of the bonded interface. Dent Mater 2008; 24: 90101.

3) Sarr M, Kane AW, Vreven J, Mine A, Van Landuyt KL, Peumans M, Lambrechts P, Van Meerbeek B, De Munck J. Microtensile bond strength and interfacial characterization of 11 contemporary adhesives bonded to bur-cut dentin. Oper Dent 2010; 35: 94-104.

4) Tay FR, Pashley DH, Yoshiyama M. Two modes of nanoleakage expression in single-step adhesives. J Dent Res 2002; 81: 472 476.

5) van Dijken JW, Sunnegårdh-Grönberg K, Lindberg A. Clinical long-term retention of etch-and-rinse and self-etch adhesive systems in non-carious cervical lesions: a 13 years evaluation. Dent Mater 2007; 23: 1101-1107.

6) Toledano M, Proenca JP, Erhardt MC, Osorio E, Aguilera FS, Osorio R, Aguilera FS, Osorio R, Tay FR. Increases in dentin- 
bond strength if doubling application time of an acetonecontaining one-step adhesive. Oper Dent 2007; 32: 133-137.

7) Zhang Y, Wang Y. Effect of application mode on interfacial morphology and chemistry between dentine and self-etch adhesives. J Dent 2013; 41: 231-240.

8) do Amaral RC, Stanislawczuk R, Zander-Grande C, Gagler D, Reis A, Loguercio AD. Bond strength and quality of the hybrid layer of one-step self-etch adhesives applied with agitation on dentin. Oper Dent 2010; 35: 211-219.

9) Miyazaki M, Platt JA, Onose H, Moore BK. Influence of dentin primer application methods on dentin bond strength. Oper Dent 1996; 21: 167-172.

10) Lee J, Jang KT, Kim JW, Lee SH, Hahn SH, Kim CC. Effect of ultrasonic vibration on dentin bond strength and resin infiltration. Am J Dent 2003; 16: 404-408.

11) Bagis B, Turkaslan S, Vallittu PK, Lassila LV. Effect of high frequency ultrasonic agitation on the bond strength of selfetching adhesives. J Adhes Dent 2009; 11: 369-374.

12) Bagis B, Turkarslan S, Tezvergil-Mutluay A, Uctasli S, Vallittu PK, Lassila LV. Effect of ultrasonic agitation on bond strength of self-etching adhesives to dentin. J Adhes Dent 2008; 10: 441-445.

13) Hanabusa M, Mine A, Kuboki T, Momoi Y, Van Ende A, Van Meerbeek B, De Munck J. Bonding effectiveness of a new 'multi-mode'adhesive to enamel and dentine. J Dent 2012; 40: 475-484.

14) Yoshihara K, Yoshida $Y$, Hayakawa S, Nagaoka N, Irie M, Ogawa T, Van Landuyt KL, Osaka A, Suzuki K, Minagi S, Van Meerbeek B. Nanolayering of phosphoric acid ester monomer on enamel and dentin. Acta Biomater 2011; 7: 3187 3195.

15) Yoshida Y, Yoshihara K, Nagaoka N, Hayakawa S, Torii Y, Ogawa T, Osaka A, Meerbeek BV. Self-assembled nanolayering at the adhesive interface. J Dent Res 2012; 91: 376381.

16) Yoshihara K, Yoshida Y, Nagaoka N, Fukegawa D, Hayakawa S, Mine A, Nakamura M, Minagi S, Osaka A, Suzuki K, Van Meerbeek B. Nano-controlled molecular interaction at adhesive interfaces for hard tissue reconstruction. Acta Biomater 2010; 6: 3573-3582.

17) Van Meerbeek B, Yoshihara K, Yoshida Y, Mine A, J De Munck, Van Landuyt KL. State of the art of self-etch adhesives. Dent Mater 2011; 27: 17-28.

18) Takahashi H. Effect of calcium salt of 10-methacryloyloxydecyl dihydrogen phosphate produced on the bond durability of one-step self-etch adhesive. Dent Mater J 2014; 33: 394-401.

19) Van Landuyt KL, Snauwaert J, De Munck J, Peumans M, Yoshida Y, Poitevin A, Coutinho E, Suzuki K, Lambrechts P, Van Meerbeek B. Systematic review of the chemical composition of contemporary dental adhesives. Biomaterials 2007; 28: 3757-3785.

20) Van Landuyt KL, De Munck J, Snauwaert J, Coutinho E, Poitevin A, Yoshida Y, Inoue S, Peumans M, Suzuki K, Lambrechts P, Van Meerbeek B. Monomer-solvent phase separation in one-step self-etch adhesives. J Dent Res 2005; 84: $183-188$.

21) Van Landuyt KL, Snauwaert J, Peumans M, De Munck J, Lambrechts P, Van Meerbeek B. The role of HEMA in onestep self-etch adhesives. Dent Mater 2008; 24: 1412-1419.

22) Yiu CK, Pashley EL, Hiraishi N, King NM, Goracci C, Ferrari M, Carvalho RM, Pashley DH, Tay FR. Solvent and water retention in dental adhesive blends after evaporation. Biomaterials 2005; 26: 6863-6872.

23) Torkabadi S, Nakajima M, Ikeda M, Foxton RM, Tagami J. Bonding durability of HEMA-free and HEMA-containing onestep adhesives to dentine surrounded by bonded enamel. J Dent 2008; 36: 80-86.

24) Tay FR, Moulding KM, Pashley DH. Distribution of nanofillers from a simplified-step adhesive in acid-conditioned dentin. J Adhes Dent 1999; 1: 103-117.

25) Reis A, Leite TM, Matte K, Michels R, Amaral RC, Geraldeli $\mathrm{S}$, Loguercio AD. Improving clinical retention of one-step selfetching adhesive systems with an additional hydrophobic adhesive layer. J Am Dent Assoc 2009; 140: 877-885.

26) Muñoz MA, Luque I, V Hass V, Reis A, Loguercio AD, Bombarda NH. Immediate bonding properties of universal adhesives to dentine. J Dent 2013; 41: 404-411.

27) Perdigão J, Kose C, Mena-Serrano A, Paula E De, Tay L, Reis A, Loguercio AD. A new universal simplified adhesive: 18month clinical evaluation. Oper Dent 2014; 39: 113-127.

28) De Munck J, Ermis RB, Koshiro K, Inoue S, Ikeda T, Sano H, Van Landuyt KL, Van Meerbeek B. NaOCl degradation of a HEMA-free all-in-one adhesive bonded to enamel and dentin following two air-blowing techniques. J Dent 2007; 35: 7483.

29) Jang J, Lee M, Woo S, Lee C, Lee J, Kim D. Comparative study of the dentin bond strength of a new universal adhesive. Dent Mater J 2016; 35: 606-612. 